\title{
Track Drawings of Graphs with Constant Queue Number
}

\author{
Emilio Di Giacomo ${ }^{1}$ and Henk Meijer ${ }^{2}$ \\ 1 Università degli Studi di Perugia, Perugia, Italy. \\ digiacomo@diei.unipg.it \\ 2 Queen's University, Kingston, Ontario, Canada. \\ henk@cs.queensu.ca
}

\begin{abstract}
A $k$-track drawing is a crossing-free $3 D$ straight-line drawing of a graph $G$ on a set of $k$ parallel lines called tracks. The minimum value of $k$ for which $G$ admits a $k$-track drawing is called the track number of $G$. In [9] it is proved that every graph from a proper minor closed family has constant track number if and only if it has constant queue number. In this paper we study the track number of well-known families of graphs with small queue number. For these families we show upper bounds and lower bounds on the track number that significantly improve previous results in the literature. Linear time algorithms that compute track drawings of these graphs are also presented and their volume complexity is discussed.
\end{abstract}

\section{Introduction and Overview}

The problem of computing a drawing of a graph with small area/volume has received a lot of attention in the graph drawing literature during the last decade.

Felsner et al. [5] initiated the study of integer grids consisting of parallel grid lines, called tracks. In particular, they focus on the box and the 3-prism. A box is a grid consisting of four parallel lines, one grid unit apart from each other and a 3-prism uses three non-coplanar parallel lines. It is shown that all outerplanar graphs can be drawn on a 3-prism where the length of the lines is $O(n)$. This result gives the first algorithm to compute a crossing-free straightline 3D drawing with linear volume for a non-trivial family of planar graphs. Moreover it is shown that there exist planar graphs that cannot be drawn on the prism and that even a box does not support all planar graphs.

Dujmovic et al. 2] show that if a graph $G$ admits a drawing $\Gamma$ on a a grid $\phi$ consisting of a constant number of parallel lines, then $G$ has a linear volume upper bound. This result suggests that the focus of the research should be on minimizing the number of tracks in a restricted integer grid, independent of the length of the tracks themselves. The track number $\operatorname{tn}(G)$ of a graph $G$ is the minimum number of tracks that is required to compute such a drawing.

\footnotetext{
* Research partially supported by "Progetto ALINWEB: Algoritmica per Internet e per il Web", MIUR - PRIN. We thank Giuseppe Liotta for useful discussions on the subject of this paper.
} 
Wood [9] shows a relationship between $\operatorname{tn}(G)$ and another well-studied graph parameter, the queue number $q n(G)$ (i.e. the minimum number of queues in a queue layout of $G$ [8]). He proves that every graph from a proper minor closed family has constant track number if and only if it has constant queue number. By the result of Wood all families of graphs whose queue number is known to be constant (for example series-parallel graphs, Halin graphs, Benes networks, arched leveled planar graphs, X-trees, unicyclic graphs), have a three dimensional straight-line grid drawing with linear volume. A recent result by Dujmović and Wood 4 s shows that linear volume can also be achieved for graphs with bounded tree width. We observe however that value of the track number (and hence of the volume) deriving from the results above are often very large. In [3] it is shown that $\operatorname{tn}(G) \leq c(2 q n(G)+1)^{c-1}$ where $c$ is the star chromatic number of $G$ ( $c \geq 3$ for any graph). Therefore, even for the (apparently innocent) family of planar graphs whose queue number is 1 we obtain an upper bound for the track number of at least 27 and by using the technique in 2] a drawing with a bound on the volume of $54 \times 59 \times 59\left\lceil\frac{n}{27}\right\rceil$. From the observation above it is natural to ask whether it is possible to reduce the bounds on track number and volume for graphs with constant queue number.

In this paper we present new lower and upper bounds on the track number (and hence on the volume) of some families of graphs that are known to have constant queue number. Our main contributions can be listed as follows.

- The family of graphs with queue number one (i.e. arched leveled planar graphs) are proved to have track number at least four and at most five. A drawing algorithm is presented for arched leveled planar graphs that gives a volume bound of $3 \times 3 \times n$.

- The track number of $X$-trees (that have queue number 2 ) is proved to be three and a volume bound of $2 \times 2 \times \frac{4(n+1)}{7}$.

- A lower bound of three and an upper bound of four is presented for Halin Graphs. A volume bound of $2 \times 2 \times n$ is also shown.

The drawing algorithm described in order to prove the above results all have $O(n)$ time complexity and use integer arithmetic.

The remainder of of the paper is organized as follows. In Section 2 some definitions and results about track and queue layout are recalled. The bounds on the track number and on the volume of arched leveled planar graphs are proved in Section 3. The track number of $X$-trees and Halin Graphs is studied in Section 4. For reasons of space some detail are omitted.

\section{Preliminaries}

Let $G=(V, E)$ be a graph. A track assignment of $G$ consists of a partition $\left\{t_{i} \mid i \in I \subseteq \mathbb{N}\right\}$ of $V$, and of a total ordering $<_{i}$ of the vertices in each set $t_{i}$. Each set $t_{i}$ is called a track-set. An overlap in a track assignment consists of three vertices $u, v$, and $w$ such that they are in the same track-set $t_{i}$, there exists 
the edge $(u, w)$ and $u<_{i} v<_{i} w$. An $X$-crossing in a track assignment consists of two edges $\left(u_{0}, v_{0}\right)$ and $\left(u_{1}, v_{1}\right)$ such that $u_{0}$ and $u_{1}$ are on the same track-set $t_{i}, v_{0}$ and $v_{1}$ are on another track-set $t_{j}(i \neq j)$ with $u_{0}<_{i} u_{1}$ and $v_{1}<_{j} v_{0}$. Figure $1(\mathrm{a})$ shows an example of track assignment for a graph $G$. Vertices $v_{1}, v_{5}$ and $v_{2}$ form an overlap, as well as vertices $v_{3}, v_{6}$ and $v_{4}$. Edges $\left(v_{5}, v_{4}\right),\left(v_{2}, v_{3}\right)$ form an $X$-crossing. Another $X$-crossing is formed by edges $\left(v_{6}, v_{8}\right)$ and $\left(v_{4}, v_{7}\right)$.

A track layout is a track assignment with no overlaps and no $X$-crossings. Figure 1(b) shows an example of a track layout of the graph of Figure 1(a) A track layout with $k$ track-sets is also called a $k$-track layout. The track number of a graph $G$, denoted by $\operatorname{tn}(G)$, is the minimum $k$ such that $G$ has a $k$-track layout 1 . A set of $k$ track-sets is also called a $k$-prism.

In the rest of the paper a track layout will be specified by assigning to each vertex $v$ two numbers: $\operatorname{track}(v)$ is an integer that denotes the index of the trackset containing $v$; order $(v)$ is an integer that denotes the ordering of $v$ in $\operatorname{track}(v)$. We say that $u<_{i} v$ if $\operatorname{track}(u)=\operatorname{track}(v)=i$ and $\operatorname{order}(u)<\operatorname{order}(v)$. We shall sometimes simplify the notation and write $u<v$ instead of $u<_{i} v$.

A track is a set of $3 \mathrm{D}$ grid points on straight line of infinite length. We always assume that a track is parallel to the $x$-axis, thus a track is the set of all the grid points having the same $y$ - and $z$-coordinate. We denote as $(x, Y, Z)$ a track whose points have $y$-coordinate $Y$ and $z$-coordinate $Z$. A track drawing of a graph $G$ on $k$ tracks, also called $k$-track drawing, is a 3D straight-line crossing-free grid drawing of $G$ such that each vertex of $G$ is drawn on one of $k$ tracks. The drawing in Figure 1(c) is a track drawing of the graph of Figure 1(a) The $k$ tracks of a $k$-track drawing will be denoted as $T_{0}, T_{1}, \ldots, T_{k-1}$. A strip $\sigma_{i j}$ is the portion of plane delimited by tracks $T_{i}$ and $T_{j}$. A strip is assumed to be an open set. Two strips $\sigma_{i j}$ and $\sigma_{h l}$ are called crossing strips if they cross each other. If a drawing of a graph is contained in an axis-aligned box with side lengths $X-1, Y-1$ and $Z-1$ then we say that the volume of the drawing is $X \times Y \times Z$.

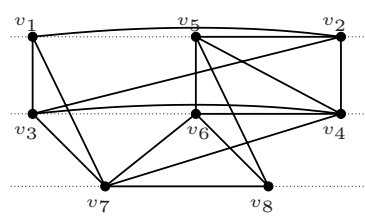

(a)

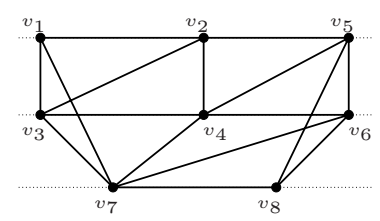

(b)

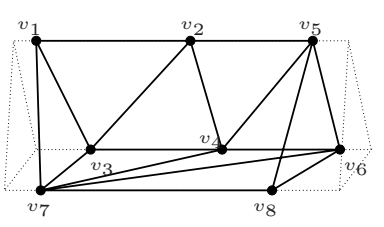

(c)

Fig. 1. (a) A track assignment of a graph $G$. (c) A track layout of $G$. (d) A track drawing of $G$.

${ }^{1}$ The concepts of track assignment and track layout are introduced also in [2], where it is assumed that no edge has both vertices in a same track-set. 
In 2 a general technique is described for computing a track drawing of a graph $G$ from a track layout of $G$. In order to reduce the volume of the drawing we use a different technique to obtain a track drawing from a track layout. The obtained drawing have neither overlaps nor $X$-crossings, because there is no overlap and no $X$-crossing in a track layout. The only possible crossings are crossings between two edges on a pair of crossing strips. Let $e_{0}=\left(u_{0}, v_{0}\right)$ and $e_{1}=\left(u_{1}, v_{1}\right)$ be two edges of $G$ on two crossing strips $\sigma_{i j}$ and $\sigma_{h l}$. If $e_{0}$ and $e_{1}$ cross each other, then the four points representing $u_{0}, v_{0}, u_{1}$ and $v_{1}$ are co-planar, i.e. the following equation is satisfied:

$$
\left|\begin{array}{cccc}
1 & 1 & 1 & 1 \\
x\left(u_{0}\right) & x\left(u_{1}\right) & x\left(v_{0}\right) & x\left(v_{1}\right) \\
y\left(u_{0}\right) & y\left(u_{1}\right) & y\left(v_{0}\right) & y\left(v_{1}\right) \\
z\left(u_{0}\right) & z\left(u_{1}\right) & z\left(v_{0}\right) & z\left(v_{1}\right)
\end{array}\right|=0
$$

The substitution of the $y$ - and $z$-coordinates of each vertex in the equation above gives a condition on the $x$-coordinates of the vertices that must be satisfied in order to have a crossings between $e_{0}$ and $e_{1}$. Thus it is sufficient to prove that the equation has no solution in order to prove that $e_{0}$ and $e_{1}$ do not cross each other. We call this equation co-planarity equation of $e_{0}$ and $e_{1}$.

A queue layout [8] of a graph $G$ consists of a linear ordering $\lambda$ of the vertices of $G$, and a partition of the edges of $G$ into queues, such that no two edges in the same queue are nested with respect to $\lambda$. In other words there are no edges $e_{0}=\left(u_{0}, v_{0}\right)$ and $e_{1}=\left(u_{1}, v_{1}\right)$ such that $e_{0}$ and $e_{1}$ are assigned to the same queue and $u_{0}<u_{1}<v_{1}<v_{0}$ in $\lambda$. A queue layout with $q$ queues is also called a q-queue layout. The queue number of a graph $G$, denoted by $q n(G)$, is the minimum $q$ such that $G$ has a $q$-queue layout.

The following theorem 3 relates the track number of a graph to the queue number of a graph.

Theorem 1. [3] Let $G$ be a graph with star chromatic number $\chi_{s t}(G) \leq c$, and queue number $q n(G) \leq q$. Then $G$ has a t-track layout, where $t \leq c(2 q+1)^{c-1}$.

\section{Graphs with $q n=1$}

Graphs with queue number equal to 1 are characterized in 8], where it is shown that they are planar graphs and admit a leveled planar embedding. For this reason they are called arched leveled planar graphs. We first give a lower bound on the track number and then we present an upper bound that improves the result in 9]. We start with a basic lemma that is used to prove the lower bound.

Lemma 1. Let $G_{0}$ be the graph in Figure 2(a), Then in any 3-track layout, vertices $u$ and $v$ are in different track-sets, and at least one of the vertices $w_{i}$ ( $i=0, \ldots, 3)$ is on the third track-set.

Lemma 2. Let $G$ be the graph in Figure 2(b). Then $q n(G)=1$ and $\operatorname{tn}(G) \geq 4$. 


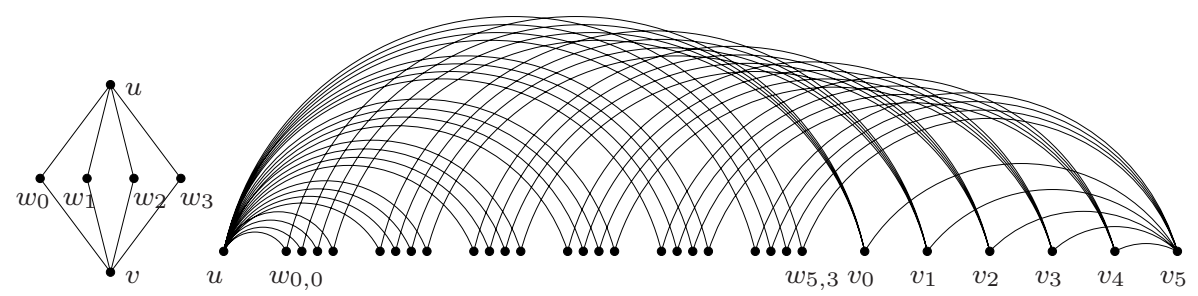

(a)

(b)

Fig. 2. (a) Graph $G_{0}$ of Lemma 1 (b) Graph $G$ with $q n(G)=1$ and $\operatorname{tn}(G)=4$.

Proof. The proof that $G$ has queue number 1 follows from Figure 2(b) where a 1-queue layout of $G$ is given. We prove that it does not admit a track layout on 3 track-sets. Suppose for a contradiction that there exists a 3-track layout of $G$ with track-sets $t_{0}, t_{1}$ and $t_{2}$, and let $t_{0}$ be the track-set containing $u$. By Lemma 1 vertices $v_{i}(0 \leq i \leq 5)$ must be in a track-set different from $t_{0}$. Let $t_{1}$ be the track-set containing $v_{5}$. Two cases are possible:

1. There exist three distinct vertices $v_{i}, v_{j}$ and $v_{k}(0 \leq i, j, k \leq 4)$ that are in $t_{1}$. In this case two of the three edges $\left(v_{5}, v_{i}\right),\left(v_{5}, v_{j}\right)$, and $\left(v_{5}, v_{k}\right)$ form an overlap.

2. There exist three distinct vertices $v_{i}, v_{j}$ and $v_{k}$, that are in $t_{2}$. By Lemma 1 there exist three vertices $w_{i, a}, w_{j, b}$ and $w_{k, c}(0 \leq a, b, c \leq 3)$ in $t_{1}$ adjacent to $u$ and to $v_{i}, v_{j}$ and $v_{k}$ respectively. Assume without loss of generality that $v_{i}<v_{j}<v_{k}$ in $t_{2}$. It follows that $w_{i, a}<w_{j, b}<w_{k, c}$ in $t_{1}$, because otherwise there would be an $X$-crossing between edges $\left(v_{i}, w_{i, a}\right),\left(v_{j}, w_{j, b}\right)$ and $\left(v_{k}, w_{k, c}\right)$. If $v_{5}<w_{i, a}$ then edges $\left(v_{5}, v_{j}\right)$ and $\left(v_{i}, w_{i, a}\right)$ form an $X$ crossing. If $w_{i, a}<v_{5}<w_{j, b}$ then edges $\left(v_{5}, v_{k}\right)$ and $\left(v_{j}, w_{j, b}\right)$ form an $X$ crossing. If $w_{j, b}<v_{5}<w_{k, c}$ then edges $\left(v_{5}, v_{i}\right)$ and $\left(v_{j}, w_{j, b}\right)$ form an $X$ crossing. Finally, if $v_{5}>w_{k, c}$ then edges $\left(v_{5}, v_{j}\right)$ and $\left(v_{k}, w_{k, c}\right)$ form an $X$-crossing.

It follows that $\operatorname{tn}(G) \geq 4$.

In order to prove the upper bound on the track number of arched leveled planar graphs we first describe a block decomposition of a connected graph $G$ with $q n(G)=1$. Then we will describe how to assign the vertices to 5 track-sets. Suppose we have a 1-queue layout of $G$ where the linear ordering of the vertices is $\lambda=v_{0}, v_{1}, \ldots, v_{n-1}$. We say that $v_{i}<v_{j}$ if $i<j$. A vertex $v$ is called a special cut vertex if there are no edges $(u, w)$ with $u<v<w$. A block is a subset of consecutive vertices in $\lambda$. The first vertex of a block $B_{i}$ is called source vertex and is denoted as $s_{i}$. The last vertex of a block $B_{i}$ is called sink vertex and is denoted as $t_{i}$. All the other vertices are called internal vertices of $B_{i}$. The 
block decomposition is defined as follows: (i) Block $B_{0}$ consists of the vertices $v_{0}, v_{1}, \ldots, v_{j}$, where $v_{j}$ is such that there exists the edge $\left(v_{0}, v_{j}\right)$ and there is no edge $\left(v_{0}, v_{h}\right)$ with $v_{j}<v_{h}$. In other words $j$ is the largest index such that there is an edge $\left(v_{0}, v_{j}\right)$. (ii) If the sink vertex $t_{i-1}$ of block $B_{i-1}$ is not a special cut vertex then block $B_{i}$ consists of the vertices $v_{k}, v_{k+1}, \ldots, v_{j}$ with the following properties: there is an edge $\left(v_{k}, v_{j}\right) ; v_{k}<t_{i-1}$; there is no edge $\left(v_{g}, v_{h}\right)$ with $v_{g}<t_{i-1}$ and $v_{j}<v_{h}$; there is no edge $\left(v_{h}, v_{j}\right)$ with $v_{h}<v_{k}$. In other words $j$ is the largest index such that there is an edge from a vertex smaller than $t_{i-1}$ to $v_{j}$ and $k$ is the smallest index such that there is an edge $\left(v_{k}, v_{j}\right)$. (iii) If the sink vertex $t_{i-1}$ of block $B_{i-1}$ is a special cut vertex then block $B_{i}$ consists of the vertices $v_{k}, v_{k+1}, \ldots, v_{j}$, where $v_{k}=t_{i-1}$, and where $v_{j}$ is such that there exists an edge $\left(t_{i-1}, v_{j}\right)$ and there is no edge $\left(t_{i-1}, v_{h}\right)$ with $h>j$. In other words $j$ is the largest index such that there is an edge from $t_{i-1}$ to $v_{j}$.

An example of a block decomposition is illustrated in Figure 3 .

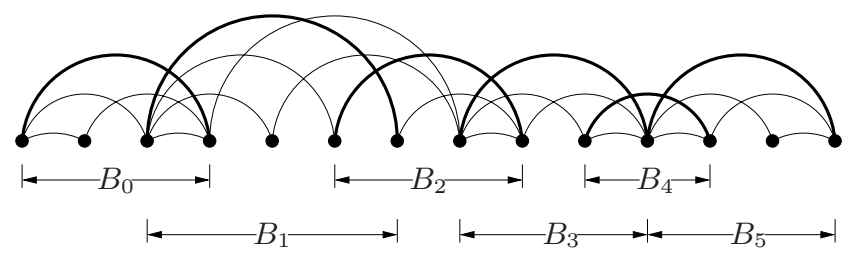

Fig. 3. A block decomposition of an arched leveled planar graph.

Algorithm 1 computes a 5-track layout of an arched leveled planar graph $G$.

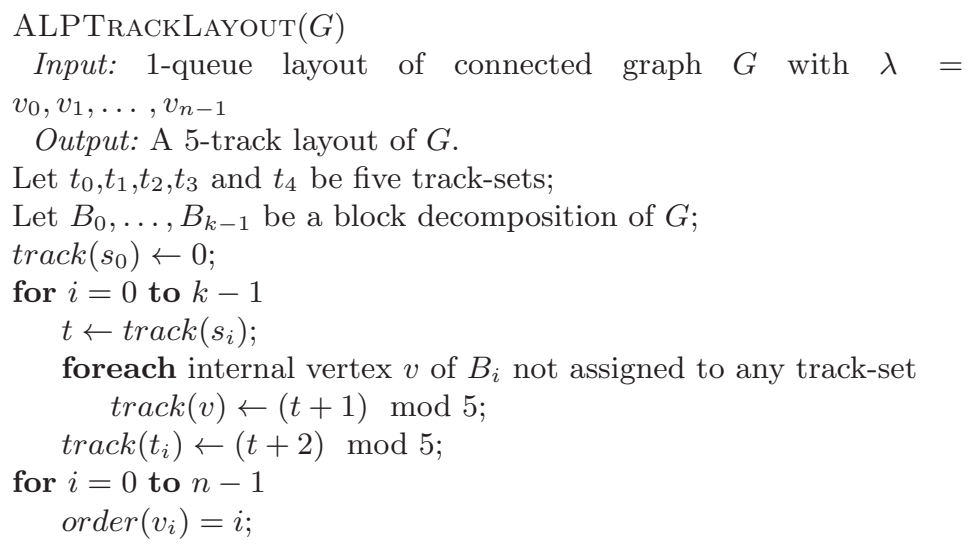

Algorithm 1: Algorithm ALPTrackLayout() 
Lemma 3. Let $G$ be a connected graph with $q n(G)=1$. Let $\lambda=v_{0}, v_{1}, \ldots, v_{n-1}$ be the linear ordering of a 1-queue layout of $G$. The track assignment computed by Algorithm 1 is such that for all edges $\left(v_{g}, v_{h}\right)$ with $g<h$, either $\operatorname{track}\left(v_{h}\right)=$ $\left(\operatorname{track}\left(v_{g}\right)+1\right) \bmod 5$ or $\operatorname{track}\left(v_{h}\right)=\left(\operatorname{track}\left(v_{g}\right)+2\right) \bmod 5$.

Lemma 4. Let $G$ be a graph with $q n(G)=1$, then $\operatorname{tn}(G) \leq 5$.

Proof. We first assume that $G$ is connected. Suppose we have a 1-queue layout of $G$ with $\lambda=v_{0}, v_{1}, \ldots, v_{n-1}$. We prove that Algorithm 1 computes a track layout of $G$. We have no overlaps since there are no edges $\left(v_{i}, v_{j}\right)$ with $\operatorname{track}\left(v_{i}\right)=$ $\operatorname{track}\left(v_{j}\right)$. Also we have no $X$-crossing. Consider two edges $\left(v_{g}, v_{h}\right)$ and $\left(v_{i}, v_{j}\right)$ such that $v_{g}$ and $v_{i}$ are in a track-set $a$ and $v_{h}$ and $v_{j}$ are in another track-set $b$. By Lemma 3, there are two cases: (i) if $b=(a+1) \bmod 5$ or $b=(a+2) \bmod 5$, then $v_{g}<v_{h}$ and $v_{i}<v_{j}$; (ii) if $b=(a-1) \bmod 5$ or $b=(a-2) \bmod 5$, then $v_{g}>v_{h}$ and $v_{i}>v_{j}$. Consider case (i), and assume without loss of generality that $v_{g}<v_{i}$. If $v_{j}<v_{h}$ then $v_{g}<v_{i}<v_{j}<v_{h}$ in $\lambda$, but this is not possible, because there would be two nested edges in the 1-queue layout. Hence $v_{h}<v_{j}$ and therefore there is no $X$-crossing. With analogous arguments also case (ii) can be considered.

Lemma 4 proves that every arched leveled planar graph $G$ has track number at most 5. The following theorem summarize the results above and describe how to produce a 5 -track drawing with volume $3 \times 3 \times n$.

Theorem 2. Every arched leveled planar graph has track number at most 5 and admits a 5-track drawing with volume at most $3 \times 3 \times n$ that can be computed in $O(n)$ time. Also, there exists an arched leveled planar graph $G$ such that $\operatorname{tn}(G) \geq 4$.

Proof. The bounds on the track number follow from Lemma 2 and 4 . We describe now how to compute a track drawing with volume $3 \times 3 \times n$. Consider the five tracks $(x, 2,1),(x, 0,1),(x, 0,0),(x, 2,0)$ and $(x, 1,2)$ and denote them as $T_{0}, T_{1}, T_{2}, T_{3}$, and $T_{4}$, respectively. Compute a track layout with five track-sets using Algorithm [1. Let $n_{0}, n_{1}, n_{2}, n_{3}$ and $n_{4}$ be the number of vertices in trackset $t_{0}, t_{1}, t_{2}, t_{3}$ and $t_{4}$, respectively. Draw the vertices assigned to track-set $t_{i}$ on track $T_{i}$ according to the total order defined in the track-set, so that they occupy $x$-coordinates from $\sum_{j=0}^{i-1} n_{j}$ to $\sum_{j=0}^{i} n_{j}(i=0,1,2,3,4)$. We prove that the drawing has no crossing. Overlaps and $X$-crossings are not possible, because there is no overlap and no $X$-crossing in the track layout.

A crossing is possible only between edges that are on crossing strips. There are five pairs of such strips: (1) $\sigma_{02}$ and $\sigma_{13} ;(2) \sigma_{01}$ and $\sigma_{24} ;(3) \sigma_{01}$ and $\sigma_{34}$; (4) $\sigma_{02}$ and $\sigma_{34} ;(5) \sigma_{13}$ and $\sigma_{24}$. Let $\sigma_{i j}$ and $\sigma_{h l}$ be the two crossing strips of one of the five cases above and let $e_{1}$ and $e_{2}$ be two edges on $\sigma_{i j}$ and $\sigma_{h l}$, respectively. Denote as $x_{i}, x_{j}, x_{h}$ and $x_{l}$ the $x$-coordinates of the vertices on tracks $T_{i}, T_{j}, T_{h}$ and $T_{l}$. The co-planarity equations for each of the five cases are: (1) $x_{0}+x_{2}=x_{1}+x_{3}$ (2) $x_{0}+3 x_{1}=2 x_{2}+2 x_{4}$ (3) $3 x_{0}+x_{1}=2 x_{3}+2 x_{4}$ (4) $4 x_{0}+x_{2}=3 x_{3}+2 x_{4}(5) 4 x_{1}+x_{3}=3 x_{2}+2 x_{4}$. Since $x_{0}<x_{1}<x_{2}<x_{3}<x_{4}$, none of the above equations has a solution, and therefore no crossing is possible. 
The drawing algorithm consider a vertex per time and executes a constant number of operations for each vertex. The time complexity is then $O(n)$.

Theorem 2 shows that every arched leveled planar graph admits a 5-track layout. However, there are specific classes of graphs that have queue number 1 and that admit a track layout with less than 5 track-sets. One of such class is that of square meshes. The following Theorem hold. Proof is omitted for reasons of space.

Theorem 3. Every square mesh has track number at most 3 and admits a 3track drawing with volume at most $2 \times 2 \times \frac{n+2 \sqrt{n}}{3}$ that can be computed in $O(n)$ time. Also, there exists a square mesh $G$ such that $\operatorname{tn}(G) \geq 3$.

\section{Graphs with $2 \leq q n \leq 3$}

In this section we study the track number of graphs with queue number equal to 2 or queue number greater than 2 and less than 3 . $X$-trees have queue number 2 [8]. The following theorem, whose proof is omitted for reasons of space, hold.

Theorem 4. Every $X$-tree has track number at most 3 and admits a 3-track drawing with volume at most $2 \times 2 \times \frac{4}{7}(n+1)$ that can be computed in $O(n)$ time. Also, there exists an $X$-tree $G$ such that $\operatorname{tn}(G) \geq 3$.

Halin Graphs [7] have queue number greater than 2 and less than 3 [6]. A Halin graph is a graph such that: (i) every vertex of $G$ has degree greater or equal to 3;(ii) $G$ can be decomposed into a spanning tree $T$ of $G$ and a cycle $C$ through the leaves of $T$; (iii) $G$ has a planar embedding in which $C$ is the boundary of the external face.

$T$ is called the characteristic tree of $G$ and $C$ is called the adjoint cycle of $G$. Figure 4 shows a Halin graph.

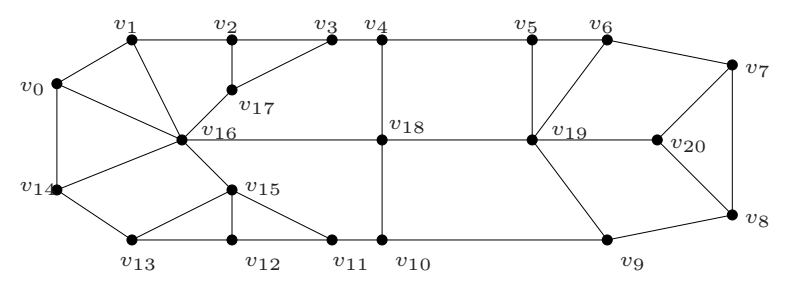

Fig. 4. A Halin graph.

It is known that a graph admits a 2-track layout only if it is outerplanar [1]. Since Halin graphs are not outerplanar (every outerplanar graph has at least on vertex of degree two) a lower bound on the track number of Halin graphs is 3 .

We now describe an algorithm to compute a 4-track layout of a Halin graph. An external path of an embedded rooted ordered tree $T$ is the path $\pi=\pi_{l} \cup \pi_{r}$, 
where $\pi_{l}$ is the path from the leftmost leaf of $T$ to the root of $T$ and $\pi_{r}$ is the path from the root of $T$ to the rightmost leaf of $T$. Let $v$ be a vertex in an external path $\pi$. If $v$ has children that are not in $\pi$ then every subtree rooted at a child of $v$ not in $\pi$ is called a dangling subtree of $\pi$.

Let $G$ be a Halin graph. Assume that $G$ is embedded in the plane such that it is planar and its adjoint cycle $C$ is the exterior face. Let $T$ be the characteristic tree of $G$. $T$ inherits its embedding from $G$. Arbitrarily choose one of the non-leaf vertices of $T$ as the root. A level decomposition of $T$ is an assignment of a level to each vertex $v$ of $T$ that is defined as follows (see Figure 5): (i) all the vertices on the external path of $T$ are given level 0 ; (ii) Let $\pi$ be an external path of level $i$. For any dangling subtree $T^{\prime}$ of $\pi$, the vertices on the external path of $T^{\prime}$ are given level $i+1$.

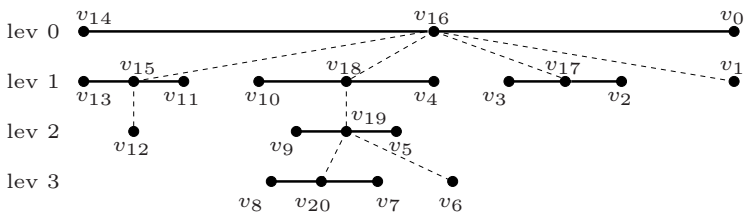

Fig. 5. A level decomposition of the characteristic tree of the Halin graph in Figure 4.

Let $\pi$ be an external path of any level. Let $T_{0}, T_{1}, \ldots, T_{h-1}$ be the dangling subtrees of $\pi$. We define a natural ordering of the dangling subtrees as follows: (i) $T_{0}, T_{1}, \ldots, T_{h-1}$ are ordered from left to right according to their parents order in $\pi$; (ii) dangling subtrees that have the same parent are ordered from left to right. A consequence of the level decomposition is the following.

Property 1. Let $G$ be a Halin graph and let $\pi$ be an external path of any level with at least one dangling subtree. Let $T_{0}, T_{1}, \ldots, T_{h-1}$ be the natural order of the dangling subtrees of $\pi$. Then in the adjoint cycle $C$ of $G$ : (i) the leftmost leaf of $\pi$ is adjacent to the leftmost leaf of $T_{0}$; (ii) the rightmost leaf of $\pi$ is adjacent to the rightmost leaf of $T_{h-1}$; (iii) the rightmost leaf of $T_{j}$ is adjacent to the leftmost leaf of $T_{j+1}(j=0, \ldots, h-2)$.

Since the embedding of $G$ is not changed when $T$ is rooted, and since the boundary of the external face of $G$ is $C$, then there exist an edge of $C$ that connects the leftmost leaf of $T$ to the rightmost leaf of $T$. The edge connecting the leftmost leaf of $T$ and the rightmost leaf of $T$ is called the long edge. Let $\pi$ be an external path without dangling subtrees; if $\pi$ consists of three vertices $u$, $v$ and $w$ in this order and $u$ and $w$ are leafs of $T$, then $u$ and $w$ are connected by an edge of $C$ and this edge is called a short edge. The long edge and the short edges are generically called overlapping edges.

We now describe an algorithm to compute a 3-track layout of a Halin graph without overlapping edges. Later we will describe how the overlapping edges can be added back to the track layout using a fourth track-set. A Halin graph after the deletion of the overlapping edges is called a reduced Halin graph. 


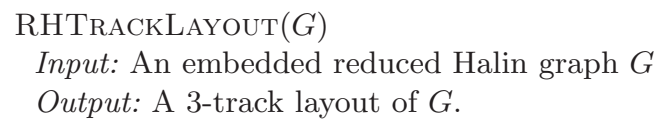

Let $T$ be the characteristic tree of $G$, rooted at any vertex $r$;

$Q \leftarrow$ new queue();

Q.enqueue $(r)$;

ord $\leftarrow 0$;

while $Q$ is not empty

$v \leftarrow$ Q.dequeue ()

if $v=r$ then $t \leftarrow 0$;

else $t \leftarrow(\operatorname{track}(\operatorname{parent}(v))+1) \bmod 3 ;$

Let $T^{\prime}$ be the subtree rooted at $v$;

Let $\pi=v_{0}, v_{1}, \ldots, v_{h-1}$ be the external path of $T^{\prime}$;

for $i=0$ to $h-1$

$\operatorname{track}\left(v_{i}\right) \leftarrow t$

$\operatorname{order}\left(v_{i}\right) \leftarrow$ ord;

ord $\leftarrow$ ord +1 ;

Let $w_{0}, w_{1}, \ldots, w_{k-1}$ be the children of $v_{i}$ not in $\pi$ ordered from left to right;

for $j=0$ to $k-1$

Q.enqueue $\left(w_{j}\right)$;

\section{Algorithm 2: Algorithm RHTrackLayout()}

Lemma 5. Let $G$ be a reduced Halin graph. Algorithm 0 computes a 3-track layout of $G$.

Proof. We prove that the track assignment computed by Algorithm 2 has no overlaps nor $X$-crossing. The edges having both vertices in a same track-set are either edges of an external path or edges of the adjoint cycle connecting leafs of the same level. The edges of the external paths do not overlap since the two vertices of each edge are consecutive in a track-set. Let $\pi$ be an external path of level $i$ and let $T_{0}, T_{1}, \ldots, T_{h-1}$ be the natural ordering of the dangling subtrees of $\pi$. By Property 1 the edges of the adjoint cycle connecting two leafs of the same level $i+1$ connect the rightmost leaf of $T_{j}$ to the leftmost leaf of $T_{j+1}$. Since Algorithm 2 lays out $T_{0}, T_{1}, \ldots, T_{h-1}$ according to their natural order, then the rightmost leaf of $T_{j}$ and the leftmost leaf of $T_{j+1}$ are consecutive in a track-set and therefore they do not overlap.

Let $e_{0}=\left(u_{0}, v_{0}\right)$ and $e_{1}=\left(u_{1}, v_{1}\right)$ be two edges having the two vertices in two different track-sets and assume that $u_{0}$ and $u_{1}$ are in the same track-set with $u_{0}<u_{1}$. Edges $e_{0}$ and $e_{1}$ are either edges connecting the roots of dangling trees to their parents or edges of the adjoint cycle connecting leafs at consecutive levels. If $u_{0}$ and $u_{1}$ are in different external path $\pi_{0}$ and $\pi_{1}$, then $u_{0}$ is adjacent to a vertex (a leaf or the root) of a dangling subtree of $\pi_{0}$ and $u_{1}$ is adjacent to a vertex (a leaf or the root) of a dangling subtree of $\pi_{1}$. The vertices of the dangling subtrees of $\pi_{0}$ precede the vertices of the dangling subtree of $\pi_{1}$ in each track-set. Therefore $v_{0}<v_{1}$ and an $X$-crossing is not possible. Assume $u_{0}$ and 
$u_{1}$ be in the same external path $\pi$. If $u_{0}$ is the leftmost vertex of $\pi$ then $v_{0}$ is the leftmost leaf of the first dangling subtree $T_{0}$ of $\pi$ (Property 1). It follows that $v_{0}<v_{1}$ and an $X$-crossing is not possible also in this case. If $u_{1}$ is the rightmost vertex of $\pi$ then $v_{1}$ is the is the rightmost leaf of the last dangling subtree $T_{h-1}$ of $\pi$ (Property 1). It follows that $v_{0}<v_{1}$ and an $X$-crossing is not possible. If $u_{0}$ is not the leftmost vertex of $\pi$ and $u_{1}$ is not the rightmost vertex of $\pi$, then they are adjacent to the roots of two dangling subtrees of $\pi$. Since $u_{0}<u_{1}$ and since the dangling subtree are laid out according to their natural order, then $v_{0}<v_{1}$ and an $X$-crossing is not possible.

Notice that the track layout of the Reduced Halin Graph is such that the vertices of each overlapping edge are both in the same track-set. The track layout of the reduced Halin graph of the graph in Figure 4 is shown in Figure 6 The long edge $\left(v_{0}, v_{14}\right)$ and the short edges $\left(v_{2}, v_{3}\right)$ and $\left(v_{7}, v_{8}\right)$ would create an overlap if considered in the track layout.

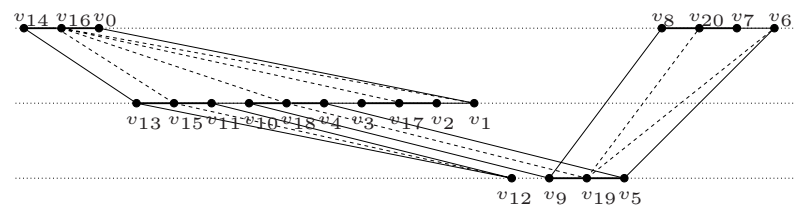

Fig. 6. A track layout of the reduced Halin graph of the graph in Figure 4

Lemma 6. Let $G$ be a Halin graph and let $G^{\prime}$ the corresponding reduced Halin graph. Let $\gamma\left(G^{\prime}\right)$ be a 3-track layout of $G^{\prime}$ computed by Algorithm 2 and let $e_{0}=\left(u_{0}, v_{0}\right)$ and $e_{1}=\left(u_{1}, v_{1}\right)$ be any pair of overlapping edges. The two edges $e_{0}$ and $e_{1}$ do not have any vertex in common, and if their vertices are in the same track-set then either $u_{0}<u_{1}<v_{0}<v_{1}$ or $u_{1}<u_{0}<v_{1}<v_{0}$.

By Lemma 6 it is easy to see that a track layout of a Halin graph can be computed from a 3-track layout of a reduced Halin graph by adding a new trackset and moving the left vertex of each overlapping edge in the new track-set.

Lemma 7. Let $G$ be a Halin graph, then $\operatorname{tn}(G) \leq 4$.

Proof. Let $\gamma(G)$ the 3-track layout of the reduced Halin graph $G^{\prime}$ of $G$ computed by Algorithm 2 Denote as $t_{0}, t_{1}$, and $t_{2}$ the three track-set used and consider a new track-set denoted as $t_{3}$. For each overlapping edge $e=(u, v)$ assume that $u<v$ and set $\operatorname{track}(u)=3$, i.e. for each overlapping edge one of the vertices is assigned to the new track-set. This change of track-set do not introduce an overlap since no edge has both vertices in track-set $t_{4}$. Also, no $X$-crossing is introduced. Namely, consider the the pair of track-sets consisting of track $t_{4}$ and of any of the other three track-sets (denote this track-set as $t_{i}$ ). The edges whose vertices are in this pair are the overlapping edges whose vertices were originally in $t_{i}$. Let $e_{0}=\left(u_{0}, v_{0}\right)$ and $e_{1}=\left(u_{1}, v_{1}\right)$ be two of these edges and assume that $u_{0}$ 
and $u_{1}$ are in track-set $t_{3}$ with $u_{0}<u_{1}$. By Lemma 6 we have $u_{0}<v_{0}<u_{1}<v_{1}$ in track-set $t_{i}$ in $\gamma\left(G^{\prime}\right)$. Since $\operatorname{order}\left(u_{0}\right)$, order $\left(v_{0}\right)$, order $\left(u_{1}\right)$, and $\operatorname{order}\left(v_{1}\right)$ are not changed, we have $u_{0}<u_{1}$ in track-set $t_{3}$ and $v_{0}<v_{1}$ in track-set $t_{i}$. So there is no $X$-crossing.

Theorem 5. Every Halin graph has track number at most 4 and admits a 4track drawing with volume at most $2 \times 2 \times n$ that can be computed in $O(n)$ time. Also, for every Halin graph $\operatorname{tn}(G) \geq 3$.

Proof. The results about the bounds on track number follows from the fact that Halin graphs are not outerplanar and by Lemma 7 We prove the result about volume. Consider the four tracks $(x, 0,0),(x, 1,0),(x, 0,1)$ and $(x, 1,1)$, and denote them as $T_{0}, T_{1}, T_{2}$, and $T_{3}$, respectively. Compute a 4 -track layout on four track-sets as described above. Let $n_{0}, n_{1}, n_{2}$ and $n_{3}$ be the number of vertices on track-set $t_{0}, t_{1}, t_{2}$ and $t_{3}$, respectively. Draw the vertices assigned to track-set $t_{i}$ on track $T_{i}$ according to the total order defined in the track-set, so that they occupy $x$-coordinates from $\sum_{j=0}^{i-1} n_{j}$ to $\sum_{j=0}^{i} n_{j}(i=0,1,2,3,4)$. We prove that the drawing has no crossing. Overlap and $X$-crossings are not possible, because there is no overlap and no $X$-crossing in the track layout. A crossing is then possible only between edges on crossing strips. There are only two such strips $\sigma_{02}$ and $\sigma_{13}$. The co-planarity equation of an edge on $\sigma_{02}$ and an edge on $\sigma_{13}$ is $x_{1}+x_{3}=x_{0}+x_{2}$. Since $x_{0}<x_{1}<x_{2}<x_{3}$ then the equation has no solution, i.e. a crossing is not possible. It follows that the drawing is a track drawing with volume $2 \times 2 \times n$. The drawing algorithm consider a vertex per time and executes a constant number of operations for each vertex. The time complexity is therefore $O(n)$.

\section{References}

1. S. Cornelsen, T. Schank, and D. Wagner. Drawing graphs on two and three lines. In Proc. GD 2002, volume 2528 of LNCS, pages 31-41. Springer-Verlag, 2002.

2. V. Dujmović, P. Morin, and D. Wood. Pathwidth and three-dimensional straight line grid drawings of graphs. In Proc. GD 2002, volume 2528 of LNCS, pages 42-53. Springer-Verlag, 2002.

3. V. Dujmović and D. Wood. New results in graph layout. Technical Report TR-03-04, School of Computer Science, Carleton University, 2003.

4. V. Dujmović and D. Wood. Tree-partitions of $k$-trees with application in graph layout. In Proc. WG 2003, LNCS. Springer-Verlag, to appear.

5. S. Felsner, G.Liotta, and S. Wismath. Straight line drawings on restricted integer grids in two and three dimensions. In Proc. GD 2001, volume 2265 of LNCS, pages 328-342. Springer-Verlag, 2001.

6. J. L. Ganley. Stack and queue layouts of Halin graphs. 1995, manuscript.

7. R. Halin. Studies in minimally connected graphs. In Combinatorial Mathematics and its Applications, pages 129-136. New York, Academic Press, 1971.

8. L. S. Heath and A. L. Rosenberg. Laying out graphs using queues. SIAM J. Computing, 21:927-958, 1992.

9. D. Wood. Queue layouts, tree-width, and three-dimensional graph drawing. In Proc. FSTTCS 2002, volume 2556 of LNCS., pages 348-359. Springer-Verlag, 2002. 